PROCEEDINGS OF THE

AMERICAN MATHEMATICAL SOCIETY

Volume 131, Number 7, Pages 2121-2127

S 0002-9939(03)06895-3

Article electronically published on February 5, 2003

\title{
ON QUASINILPOTENT OPERATORS
}

\author{
IL BONG JUNG, EUNGIL KO, AND CARL PEARCY
}

(Communicated by David R. Larson)

\begin{abstract}
In this note we modify a new technique of Enflo for producing hyperinvariant subspaces to obtain a much improved version of his "two sequences" theorem with a somewhat simpler proof. As a corollary we get a proof of the "best" theorem (due to V. Lomonosov) known about hyperinvariant subspaces for quasinilpotent operators that uses neither the Schauder-Tychonoff fixed point theorem nor the more recent techniques of Lomonosov.
\end{abstract}

\section{INTRODUCTION}

Let $\mathcal{H}$ be a separable, infinite dimensional, complex Hilbert space, and denote by $\mathcal{L}(\mathcal{H})$ the algebra of all bounded linear operators on $\mathcal{H}$ and by $\mathbf{K}=\mathbf{K}(\mathcal{H})$ the ideal of compact operators in $\mathcal{L}(\mathcal{H})$. Perhaps the first invariant-subspace theorem for operators in $\mathcal{L}(\mathcal{H})$, other than those provided by the spectral theorem for normal operators, was that every operator in $\mathbf{K}(\mathcal{H})$ has a nontrivial invariant subspace.

According to Aronszajn-Smith [3], this was proved by John von Neumann (unpublished) about 1935. Thus there has now been over a half-century of work devoted to establishing that operators in $\mathcal{L}(\mathcal{H})$ that have a nice enough relation to some compact operator have nontrivial invariant subspaces. Without attempting to be exhaustive we mention the papers of Bernstein-Robinson [4], Halmos [9], [10], Arveson-Feldman [1], Deckard-Douglas-Pearcy [7], Pearcy-Salinas [14, Lomonosov 11], 12, [13], Pearcy-Shields [15, Scott Brown [5], and, more recently, ChevreauLi-Pearcy [6], Simonic [16], Ansari-Enflo [2], and Enflo-Lomonosov 8]. Several of these works took something from previous ones, but many also added new techniques, some dramatically new (for example, the use by Lomonosov in [11] of the Schauder-Tychonoff fixed point theorem for nonlinear mappings).

In [2], a very recent new technique was introduced (and ascribed there to Enflo) for producing invariant subspaces for compact-related operators in $\mathcal{L}(\mathcal{H})$. The following old theorem of Lomonosov ([11]; cf. also [15]) was thus given in [2] a completely different proof (neither utilizing the Schauder-Tychonoff fixed point theorem nor the ideas of [12]).

Theorem 1.1. Every nonzero compact operator in $\mathcal{L}(\mathcal{H})$ has a nontrivial hyperinvariant subspace.

Received by the editors February 6, 2002.

2000 Mathematics Subject Classification. Primary 47A15.

Key words and phrases. Invariant subspaces, Enflo technique, extremal vectors.

(C)2003 American Mathematical Society 
As another consequence of this technique, Enflo in [8] obtained the following interesting "two sequences" theorem.

Theorem 1.2. Let $\mathcal{A} \subset \mathcal{L}(\mathcal{H})$ be any commutative algebra that contains a nonzero quasinilpotent operator. Then there exist sequences $\left\{s_{k}\right\}_{k=1}^{\infty}$ and $\left\{t_{k}\right\}_{k=1}^{\infty}$ in $\mathcal{H}$ that converge weakly to nonzero vectors $s_{0}$ and $t_{0}$, respectively, such that for every bounded sequence $\left\{A_{k}\right\}_{k=1}^{\infty} \subset \mathcal{A}$,

$$
\lim _{k}\left(A_{k} s_{k}, t_{k}\right)=0 \text {. }
$$

This technique of proof (from [2] and 8]) uses some "extremal vectors" in a very clever way, and, as was mentioned in 8 , is so new that most likely it will be some time before one knows whether the technique (or modifications thereof) will yield all the stronger theorems from [11] and [12] as well as perhaps some completely new results in the same direction.

The purpose of this note is to show that by modifying Enflo's new technique, a considerably better version of Theorem 1.2, with a somewhat simpler proof, can be obtained as follows.

Theorem 1.3. Suppose $Q \neq 0$ is a quasinilpotent operator in $\mathcal{L}(\mathcal{H})$ and $\{Q\}^{\prime}$ denotes the commutant of $Q$, i.e., $\{Q\}^{\prime}=\{A \in \mathcal{L}(\mathcal{H}): A Q=Q A\}$. Let $B_{0}$ be an arbitrary nonzero operator in $\{Q\}^{\prime}$ such that $B_{0} Q \neq 0$. Then there exist sequences $\left\{s_{k}\right\}_{k=1}^{\infty}$ and $\left\{t_{k}\right\}_{k=1}^{\infty}$ in $\mathcal{H}$ that converge weakly to nonzero vectors $s_{0}$ and $t_{0}$, respectively, with $B_{0} s_{0} \neq 0$, and a sequence $\left\{\beta_{k}\right\}$ of positive numbers converging to zero, such that for every doubly indexed sequence $\left\{A_{m, k}\right\}_{m, k \in \mathbb{N}}$ in the unit ball of $\{Q\}^{\prime}$, we have

$$
\left|\left(A_{m, k} s_{k}, t_{k}\right)\right|<\beta_{k}, \quad m, k \in \mathbb{N} .
$$

Also as a corollary of Theorem 1.3, the following better (than Theorem 1.1) but not so old theorem of Lomonosov [12] can be deduced.

Corollary 1.4 (12]). Suppose that $Q \neq 0$ is a quasinilpotent operator in $\mathcal{L}(\mathcal{H})$ and there exist a sequence $\left\{D_{m}\right\}_{m \in \mathbb{N}} \subset\{Q\}^{\prime}$ converging in the weak operator topology to a nonzero $\left(C\right.$ in $\left.\{Q\}^{\prime}\right)$ and a sequence $\left\{K_{m}\right\}_{m \in \mathbb{N}}$ of compact operators such that

$$
\lim _{m}\left\|D_{m}-K_{m}\right\|=0 .
$$

(In other words, in the language of [12], we suppose that $\{Q\}^{\prime}$ has the Pearcy-Salinas property.) Then $Q$ has a nontrivial hyperinvariant subspace.

In other words, this note may be considered as a first step in the direction of determining what are the best theorems that can be obtained by (modifications of) this new Enflo technique from [2] and 8]. We remark that Corollary 1.4 is the "strongest" theorem known which produces hyperinvariant subspaces for a quasinilpotent operator, so, at least in this direction, Enflo's new technique produces the "best" theorem known.

\section{Some Lemmas}

Our proof of Theorem 1.3 depends on several lemmas (essentially) from [2].

Lemma 2.1. Suppose $u$ and $v$ are nonzero vectors in $\mathcal{H}$ such that for every $z \in \mathcal{H}$, $\operatorname{Re}(u, z)<0$ implies that $\operatorname{Re}(v, z) \geq 0$. Then there exists a negative number $r_{0}$ such that $v=r_{0} u$. 
Proof. Write $v=\alpha_{0} u+w$ where $\alpha_{0} \in \mathbb{C}$ and $w$ is orthogonal to $u$. Note that if we set $z=z(\gamma, x)=\gamma u+x$, where $x$ is orthogonal to $u$ and $\operatorname{Re} \gamma<0$, then

$$
\operatorname{Re}(u, z)=\operatorname{Re}(u, \gamma u+x)=\|u\|^{2} \operatorname{Re} \gamma<0 .
$$

Thus, for all $x$ orthogonal to $u$ and for all $\gamma$ with $\operatorname{Re} \gamma<0$, we have, by hypothesis,

$$
\operatorname{Re}(v, z(\gamma, x))=\operatorname{Re}\left(\alpha_{0} u+w, \gamma u+x\right)=\|u\|^{2} \operatorname{Re}\left(\alpha_{0} \gamma\right)+\operatorname{Re}(w, x) \geq 0 .
$$

Upon fixing $\gamma$ and taking $x$ to be a large enough negative scalar multiple of $w$, we see that necessarily $w=0$ and that $\operatorname{Re}\left(\alpha_{0} \gamma\right) \geq 0$. Upon writing $\alpha_{0}=r_{0}+i s_{0}$ and $\gamma=t+i q$ where $r_{0}, s_{0}, t, q$ are real, we get that $r_{0} t-s_{0} q \geq 0$ for all $q \in \mathbb{R}$ and all $t<0$. Fixing $t$ and letting $q$ run we get $s_{0}=0$ and then $r_{0} \leq 0$. Since $v=r_{0} u$ and $v \neq 0$, we must have $r_{0}<0$, so the proof is complete.

Lemma 2.2. Suppose $T \in \mathcal{L}(\mathcal{H})$ and has dense range. Suppose also that $x_{0}$ is a nonzero vector in $\mathcal{H}$ and that $\varepsilon$ satisfies $0<\varepsilon<\left\|x_{0}\right\|$. Then there exists a unique nonzero vector $y_{0}=y_{0}\left(x_{0}, \varepsilon\right)$ such that

a) $\left\|y_{0}\right\|=\inf \left\{\|y\|:\left\|T y-x_{0}\right\| \leq \varepsilon\right\}$ and

b) $\left\|T y_{0}-x_{0}\right\|=\varepsilon$.

Proof. Let $\mathcal{F}=\left\{y \in \mathcal{H}:\left\|T y-x_{0}\right\| \leq \varepsilon\right\}$. Since $T$ has dense range, clearly $\mathcal{F}$ is nonempty, and since $T$ is continuous and $\mathcal{F}$ is the inverse image under $T$ of the norm-closed ball centered at $x_{0}$ with radius $\varepsilon, \mathcal{F}$ is a norm-closed set. Moreover an easy calculation shows that $\mathcal{F}$ is a convex set. But, as is well-known, such a set has a unique vector $y_{0}$ of minimal norm. Thus a) is satisfied, and if $\left\|T y_{0}-x_{0}\right\|<\varepsilon$, then for $\delta>0$ sufficiently small, $(1-\delta) y_{0}$ would belong to $\mathcal{F}$ and have smaller norm, so b) is satisfied.

Lemma 2.3. Suppose $T, x_{0}, y_{0}$, and $\varepsilon$ are as in Lemma 2.2. Then there exists a negative number $r$ such that $T^{*}\left(T y_{0}-x_{0}\right)=r y_{0}$.

Proof. We apply Lemma 2.1 to $u=T^{*}\left(T y_{0}-x_{0}\right)$ and $v=y_{0}$. Clearly it suffices to show that $u$ and $v$ satisfy the hypotheses of Lemma 2.1 (and then set $r=1 / r_{0}$ ). Thus suppose that $z_{0}$ is any vector in $\mathcal{H}$ satisfying

$$
\operatorname{Re}(u, z)=\operatorname{Re}\left(T^{*}\left(T y_{0}-x_{0}\right), z_{0}\right)=\operatorname{Re}\left(T y_{0}-x_{0}, T z_{0}\right)<0 .
$$

It follows easily that there exists a sufficiently small interval $\left[0, t_{0}\right]$, on which the function $t \rightarrow\left\|\left(T y_{0}-x_{0}\right)+t T z_{0}\right\|^{2}$ is strictly monotone decreasing (its derivative is continuous and negative at the origin). Thus for $t \in\left(0, t_{0}\right]$ we have

$$
\left\|T\left(y_{0}+t z_{0}\right)-x_{0}\right\|<\left\|T y_{0}-x_{0}\right\|=\varepsilon .
$$

Thus for $t \in\left(0, t_{0}\right], y_{0}+t z_{0} \in \mathcal{F}$, and by the minimality of $y_{0}$, we must have

$$
\left\|y_{0}+t z_{0}\right\|^{2} \geq\left\|y_{0}\right\|^{2}, \quad t \in\left(0, t_{0}\right] .
$$

But the derivative of the function

$$
t \rightarrow\left\|y_{0}+t z_{0}\right\|^{2}
$$

is continuous and its value at the origin is $2 \operatorname{Re}\left(y_{0}, z_{0}\right)$, which must therefore satisfy $\operatorname{Re}\left(y_{0}, z_{0}\right) \geq 0$, and the lemma is proved.

Lemma 2.4. Suppose $T \in \mathcal{L}(\mathcal{H})$ is quasinilpotent with dense range, let $x_{0}$ be a nonzero vector in $\mathcal{H}$, let $\varepsilon$ satisfy $0<\varepsilon<\left\|x_{0}\right\|$, and (via Lemma 2.2) let, for each $n \in \mathbb{N}, y_{n}=y_{n}\left(\varepsilon, x_{0}\right)$ be a (nonzero) vector satisfying

a) $\left\|y_{n}\right\|=\inf \left\{\|y\|:\left\|T^{n} y-x_{0}\right\| \leq \varepsilon\right\}$ and 
b) $\left\|T^{n} y_{n}-x_{0}\right\|=\varepsilon$.

Then there exists a subsequence $\left\{y_{n_{k}}\right\}_{k=1}^{\infty}$ of the sequence $\left\{y_{n}\right\}$ satisfying

$$
\lim _{k} \frac{\left\|y_{n_{k}}\right\|}{\left\|y_{n_{k}+1}\right\|}=0
$$

Proof. Suppose, to the contrary, that there exist $t>0$ and $N_{t} \in \mathbb{N}$ such that

$$
\inf _{n \geq N_{t}} \frac{\left\|y_{n}\right\|}{\left\|y_{n+1}\right\|}=t \text {. }
$$

Then

$$
\left\|y_{N_{t}}\right\| \geq t\left\|y_{N_{t}+1}\right\| \geq t^{2}\left\|y_{N_{t}+2}\right\| \geq \cdots \geq t^{n}\left\|y_{N_{t}+n}\right\|, \quad n \in \mathbb{N} .
$$

By the minimality of $\left\|y_{N_{t}}\right\|$ from a), we have (since $\left\|T^{N_{t}+n} y_{N_{t}+n}-x_{0}\right\|=\varepsilon$ )

$$
\left\|T^{n} y_{N_{t}+n}\right\| \geq\left\|y_{N_{t}}\right\|, \quad n \in \mathbb{N} \text {. }
$$

Thus

$$
\left\|T^{n}\right\|\left\|y_{N_{t}+n}\right\| \geq\left\|y_{N_{t}}\right\| \geq t^{n}\left\|y_{N_{t}+n}\right\|, \quad n \in \mathbb{N},
$$

and hence $\left\|T^{n}\right\| \geq t^{n}, n \in \mathbb{N}$, which contradicts the fact that $\sigma(T)=\{0\}$. The result follows.

\section{Proofs of the Results}

On the basis of these lemmas, we now prove Theorem 1.3 and Corollary 1.4.

Proof of Theorem 1.3. Let $B_{0} \in\{Q\}^{\prime}$ be such that $B_{0} Q \neq 0$. If $\mathcal{M}=$ (range $Q)^{-} \neq \mathcal{H}$, then $\mathcal{M}$ is a nontrivial hyperinvariant subspace for $Q$ and the result follows by choosing nonzero vectors $s_{0} \in \mathcal{M}$ and $t_{0} \in \mathcal{M}^{\perp}$ such that $B_{0} s_{0} \neq 0$ and defining $s_{k}=s_{0}, t_{k}=t_{0}, k \in \mathbb{N}$, and $\left\{\beta_{k}\right\}$ to be an arbitrary sequence of positive numbers tending to zero. Thus we may suppose that $Q$ has dense range (which implies that each $Q^{n}$ also has dense range). Let $x_{0}$ be a nonzero vector in $\mathcal{H}$ such that $B_{0} Q x_{0}\left(=Q B_{0} x_{0}\right) \neq 0$, and let $\varepsilon$ satisfy

$$
0<\varepsilon<\min \left\{\left\|x_{0}\right\|,\left\|Q x_{0}\right\|,\left(1 /\left\|B_{0}\right\|\right)\left\|B_{0} x_{0}\right\|\right\} .
$$

For each $n \in \mathbb{N}$, let $y_{n}=y_{n}\left(\varepsilon, x_{0}\right)$ satisfy a) and b) of Lemma 2.4 (with $T=Q$ ). By Lemma 2.4, we can choose a subsequence $\left\{y_{n_{k}}\right\}$ of $\left\{y_{n}\right\}$ such that

$$
\lim _{k} \frac{\left\|y_{n_{k}}\right\|}{\left\|y_{n_{k}+1}\right\|}=0 \text {. }
$$

By dropping down to successive subsequences of $\left\{y_{n_{k}}\right\}$ we may suppose (without changing the notation accordingly), since all of the vectors $Q^{n} y_{n}, n \in \mathbb{N}$, belong to the norm-closed ball of radius $\varepsilon$ centered at $x_{0}$, that the sequence $\left\{Q^{n_{k}} y_{n_{k}}\right\}$ converges weakly to a vector $z_{0}$ and, similarly, that the sequence $\left\{Q^{n_{k}+1} y_{n_{k}+1}\right\}$ converges weakly to a vector $v_{0}$. Since norm-closed balls in $\mathcal{H}$ are weakly closed, we have $\left\|v_{0}-x_{0}\right\| \leq \varepsilon,\left\|z_{0}-x_{0}\right\| \leq \varepsilon$ (so, in particular, $\left.v_{0} \neq 0 \neq z_{0}\right),\left\|B_{0} z_{0}-B_{0} x_{0}\right\| \leq$ $\left\|B_{0}\right\| \varepsilon$, and $B_{0} z_{0} \neq 0$.

We next show that $v_{0}-x_{0} \neq 0$, which shows also (since $\mathcal{M}=\mathcal{H}$ ) that $Q^{*}\left(v_{0}-x_{0}\right) \neq 0$. By the definition of the $y_{n}$ and Lemma 2.3, we have

$$
\begin{aligned}
\varepsilon^{2} & =\left\|Q^{n_{k}+1} y_{n_{k}+1}-x_{0}\right\|^{2} \\
& =\left(y_{n_{k}+1},\left(Q^{n_{k}+1}\right)^{*}\left(Q^{n_{k}+1} y_{n_{k}+1}-x_{0}\right)\right)-\left(x_{0}, Q^{n_{k}+1} y_{n_{k}+1}-x_{0}\right) \\
& =r_{n_{k}+1}\left\|y_{n_{k}+1}\right\|^{2}-\left(x_{0}, Q^{n_{k}+1} y_{n_{k}+1}-x_{0}\right), \quad k \in \mathbb{N},
\end{aligned}
$$


where $r_{n_{k}+1}<0$ for all $k \in \mathbb{N}$. Thus

$$
-\varepsilon^{2} \geq\left(x_{0}, Q^{n_{k}+1} y_{n_{k}+1}-x_{0}\right), \quad k \in \mathbb{N},
$$

and, taking limits as $k \rightarrow \infty$, we get $-\varepsilon^{2} \geq\left(x_{0}, v_{0}-x_{0}\right)$, so $v_{0}-x_{0} \neq 0$ and $Q^{*}\left(v_{0}-x_{0}\right) \neq 0$.

Now define

$$
\begin{aligned}
s_{k} & =Q^{n_{k}} y_{n_{k}}, \\
t_{k} & =Q^{*}\left(Q^{n_{k}+1} y_{n_{k}+1}-x_{0}\right), \\
\beta_{k} & =\left\|y_{n_{k}}\right\| \varepsilon\left(\left\|x_{0}\right\|+\varepsilon\right) /\left\|y_{n_{k}+1}\right\|, \quad k \in \mathbb{N},
\end{aligned}
$$

and note that the sequences $\left\{s_{k}\right\}_{k=1}^{\infty}$ and $\left\{t_{k}\right\}_{k=1}^{\infty}$ converge weakly to the nonzero vectors $s_{0}:=z_{0}$ and $t_{0}:=Q^{*}\left(v_{0}-x_{0}\right)$, respectively, and that the sequence $\left\{\beta_{k}\right\}$ converges to zero. Next we let $\left\{A_{m, k}\right\}_{m, k \in \mathbb{N}}$ be an arbitrary doubly indexed sequence in the unit ball of $\{Q\}^{\prime}$, and we write

$$
A_{m, k} y_{n_{k}}=\alpha_{n_{k}}^{(m)} y_{n_{k}+1}+w_{n_{k}+1}^{(m)}, \quad m, k \in \mathbb{N},
$$

where $\alpha_{n_{k}}^{(m)} \in \mathbb{C}$ and $w_{n_{k}+1}^{(m)}$ is orthogonal to $y_{n_{k}+1}$ for all $m, k \in \mathbb{N}$. Note that

$$
\begin{aligned}
\left\|y_{n_{k}}\right\|^{2} & \geq\left\|A_{m, k} y_{n_{k}}\right\|^{2} \\
& =\left|\alpha_{n_{k}}^{(m)}\right|^{2}\left\|y_{n_{k}+1}\right\|^{2}+\left\|w_{n_{k}+1}^{(m)}\right\|^{2}, \quad m, k \in \mathbb{N},
\end{aligned}
$$

and thus

$$
\frac{\left\|y_{n_{k}}\right\|}{\left\|y_{n_{k}+1}\right\|} \geq\left|\alpha_{n_{k}}^{(m)}\right|, \quad m, k \in \mathbb{N} .
$$

An application of $Q^{n_{k}+1}$ to each side of (2) gives

$$
\begin{aligned}
Q A_{m, k} Q^{n_{k}} y_{n_{k}} & =Q^{n_{k}+1} A_{m, k} y_{n_{k}} \\
& =\alpha_{n_{k}}^{(m)} Q^{n_{k}+1} y_{n_{k}+1}+Q^{n_{k}+1} w_{n_{k}+1}^{(m)}, m, k \in \mathbb{N} .
\end{aligned}
$$

Upon taking the inner product of each side of (4) with $Q^{n_{k}+1} y_{n_{k}+1}-x_{0}$, we obtain

$$
\left(A_{m, k} s_{k}, t_{k}\right)=\alpha_{n_{k}}^{(m)}\left(Q^{n_{k}+1} y_{n_{k}+1}, Q^{n_{k}+1} y_{n_{k}+1}-x_{0}\right), \quad m, k \in \mathbb{N},
$$

since, by Lemma 2.3 ,

$$
\left(w_{n_{k}+1}^{(m)},\left(Q^{n_{k}+1}\right)^{*}\left(Q^{n_{k}+1} y_{n_{k}+1}-x_{0}\right)\right)=\left(w_{n_{k}+1}^{(m)}, r_{n_{k}+1} y_{n_{k}+1}\right)=0, \quad m, k \in \mathbb{N} .
$$

Moreover, since

$$
\left|\left(Q^{n_{k}+1} y_{n_{k}+1}, Q^{n_{k}+1} y_{n_{k}+1}-x_{0}\right)\right| \leq\left(\left\|x_{0}\right\|+\varepsilon\right) \varepsilon, \quad k \in \mathbb{N},
$$

we have from the definition of $\beta_{k},(3)$, and (5) that

$$
\left|\left(A_{m, k} s_{k}, t_{k}\right)\right| \leq \beta_{k}, \quad m, k \in \mathbb{N} .
$$

Since we saw earlier that $B_{0} s_{0}=B_{0} z_{0} \neq 0$, the theorem is proved.

Proof of Corollary 1.4. We may suppose, without loss of generality, that $Q$ is a quasiaffinity (otherwise $\operatorname{ker} Q$ or (range $Q)^{-}$is a nontrivial hyperinvariant subspace for $Q$ ). Thus $C Q \neq 0$, and we set $B_{0}$ of Theorem 1.3 equal to $C$. Now let the sequences $\left\{s_{k}\right\},\left\{t_{k}\right\}$, and $\left\{\beta_{k}\right\}$ be as in Theorem 1.3, with $\left\{s_{k}\right\}$ and $\left\{t_{k}\right\}$ having nonzero weak limits $s_{0}$ and $t_{0}$, respectively. Also let $A_{0}$ be an arbitrary operator in the unit ball of $\{Q\}^{\prime}$ such that $A_{0} C \neq 0$. We will show that $\left(A_{0} C s_{0}, t_{0}\right)=0$, and therefore that $\mathcal{M}=\left(\{Q\}^{\prime} C s_{0}\right)^{-}$is the desired nontrivial hyperinvariant subspace 
for $Q$. (Note that $0 \neq C s_{0} \in \mathcal{M}$ and that $t_{0}$ is orthogonal to $\mathcal{M}$.) We may suppose, without loss of generality, that the sequence $\left\{D_{m}\right\}$ lies in the unit ball of $\{Q\}^{\prime}$. Define the doubly indexed sequence $\left\{A_{m, k}\right\}_{m, k \in \mathbb{N}}$ by $A_{m, k}=A_{0} D_{m}, m, k \in \mathbb{N}$. Then, from Theorem 1.3, we know that

$$
\left|\left(A_{0} D_{m} s_{k}, t_{k}\right)\right|<\beta_{k}, \quad m, k \in \mathbb{N} .
$$

Now let $\eta>0$ be given and note that (since $\left\{K_{m}\right\}$ tends to $C$ in the weak operator topology) it suffices to find $M_{\eta}>0$ such that

$$
\left|\left(A_{0} K_{m} s_{0}, t_{0}\right)\right| \leq \eta, \quad m \geq M_{\eta} .
$$

Choose $K>0$ such that for $k \geq K, \beta_{k}<\eta / 2$, and, by (1), choose $M_{\eta}>0$ such that

$$
\left\|D_{m}-K_{m}\right\|<\eta /\left\{2\left\|A_{0}\right\|\left(\sup _{k}\left\|s_{k}\right\|\left\|t_{k}\right\|\right)\right\}, \quad m \geq M_{\eta} .
$$

Then, via (6) and (8),

$$
\begin{aligned}
\left|\left(A_{0} K_{m} s_{k}, t_{k}\right)\right| \leq & \left|\left(A_{0} D_{m} s_{k}, t_{k}\right)\right| \\
& +\left|\left(A_{0}\left(K_{m}-D_{m}\right) s_{k}, t_{k}\right)\right|<\eta, \quad m \geq M_{\eta}, k \geq K .
\end{aligned}
$$

Fix an arbitrary $m_{0} \geq M_{\eta}$, and note that since $\left\{s_{k}\right\}$ tends weakly to $s_{0}$ and $A_{0} K_{m_{0}}$ is compact, we obtain

$$
\lim _{k}\left\|A_{0} K_{m_{0}} s_{k}-A_{0} K_{m_{0}} s_{0}\right\|=0 .
$$

Moreover, since $\left\{t_{k}\right\}$ tends weakly to $t_{0}$, we get from (9), (10), and a short calculation, that

$$
\left|\left(A_{0} K_{m_{0}} s_{0}, t_{0}\right)\right|=\lim _{k}\left|\left(A_{0} K_{m_{0}} s_{k}, t_{k}\right)\right| \leq \eta, \quad m_{0} \geq M_{\eta},
$$

which establishes (7) and completes the proof.

\section{ACKNowledgement}

The first and second authors were supported by KOSEF 2000-1-10100-002-3. The third author gratefully acknowledges the support of the National Science Foundation.

\section{REFERENCES}

[1] W. Arveson and J. Feldman, A note on invariant subspaces, Michigan Math. J. 15(1968), 61-64. MR 36:6969

[2] S. Ansari and P. Enflo, Extremal vectors and invariant subspaces, Trans. Amer. Math. Soc. 350(1998), 539-558. MR 98d:47019

[3] N. Aronszajn and K. Smith, Invariant subspaces of completely continuous operators, Ann. of Math. 60(1954), 345-350. MR 16:488b

[4] A. Bernstein and A. Robinson, Solution of an invariant subspace problem of K.T. Smith and P.R. Halmos, Pacific J. Math. 16(1966), 421-431. MR 33:1724

[5] S. Brown, Hyponormal operators with thick spectrum have invariant subspaces, Ann. of Math. 125(1987), 93-103. MR 88c:47010

[6] B. Chevreau, W. Li, and C. Pearcy, A new Lomonosov lemma, J. Operator Theory 40(1998), 409-417. MR 2000b:47014

[7] D. Deckard, R. Douglas, and C. Pearcy, On invariant subspaces of quasitriangular operators, Amer. J. Math. 9(1969), 637-647. MR 41:859

[8] P. Enflo and V. Lomonosov, Some aspects of the invariant subspace problem, preprint.

[9] P. Halmos, Invariant subspaces of polynomially compact operators, Pacific J. Math. 16(1966), 433-437. MR 33:1725

[10] _ Q Quasitriangular operators, Acta Sci. Math.(Szeged) 29(1968), 283-293. MR 38:2627. 
[11] V. Lomonosov, On invariant subspaces of families of operators commuting with a completely continuous operator (in Russian), Funkcional Anal. i Prilozen 7(1973), 55-56. MR 54:8319]

[12] _ An extension of Burnside's theorem to infinite dimensional spaces, Israel J. Math. 75(1991), 329-339. MR 93h:47007

[13] - On real invariant subspaces of bounded operators with compact imaginary part, Proc. Amer. Math. Soc. 115(1992), 775-777. MR 92i:47003

[14] C. Pearcy and N. Salinas, An invariant subspace theorem, Michigan Math. J. 20(1973), 21-31. MR 47:5623

[15] C. Pearcy and A. Shields, A survey of the Lomonosov technique in the theory of invariant subspaces, Topics in Operator Theory, Amer. Math. Soc. Surveys, No. 13(1974), 219-229. MR 50:8113

[16] A. Simonic, An extension of Lomonosov's techniques to non-compact operators, Trans. Amer. Math. Soc. 348(1996), 975-995. MR 96j:47005

Department of Mathematics, Kyungpook National University, Taegu 702-701, Korea

E-mail address: ibjung@kyungpook.ac.kr

Department of Mathematics, Ewha Women's University, Seoul 120-750, Korea

E-mail address: eiko@mm.ewha.ac.kr

Department of Mathematics, Texas A\&M University, College Station, Texas 77843

E-mail address: pearcy@math.tamu.edu 